\title{
Long-term outcomes of cardiac resynchronization therapy are worse in patients who require atrioventricular junction ablation for atrial fibrillation than in those with sinus rhythm
}

Ewa Jędrzejczyk-Patej, Radosław Lenarczyk, Patrycja Pruszkowska, Oskar Kowalski, Michał Mazurek, Adam Sokal, Joanna Boidol, Aleksandra Woźniak, Sławomir Pluta, Mariola Szulik, Agnieszka Liberska, Zbigniew Kalarus

Department of Cardiology, Congenital Heart Disease and Electrotherapy, Medical University of Silesia, Silesian Center for Heart Diseases, Zabrze, Poland

\begin{abstract}
Background: The aim of the study was to assess the impact of atrial fibrillation (AF) with and without the need for atrioventricular junction (AVJ) ablation on outcomes in patients undergoing cardiac resynchronization therapy (CRT).
\end{abstract}

Methods: A single center cohort of 200 consecutive CRT patients was divided into three groups: 1) AF with CRT pacing < $95 \%$ in which AVJ ablation was performed ( $A F-A B L$, $n=40$; 20\%), 2) AF without the need for AVJ ablation (AF-nonABL, $n=40 ; 20 \%), 3$ ) sinus rhythm (SR, $n=120 ; 60 \%)$. All patients were assessed before CRT implantation and at 6-month follow-up. Positive clinical response to CRT was considered alive status without the need for heart transplantation and improvement $\geq 1$ NYHA after 6 months. The comparative analysis among all study groups with respect to response-rate and long-term survival was performed.

Results: The 6-month response-rate in both $A F-A B L$ and $A F-n o n A B L$ was significantly lower than in $S R$ (52.5 and 50 vs.77.5\%, respectively; both $p<0.017$ ), though there were no differences in baseline characteristics among study groups apart from higher baseline $N T$-proBNP levels in AF-ABL. However, after adjustment for this confounder, and despite optimal CRT pacing burden in study groups, the remote all-cause mortality during median follow-up of 36.1 months was significantly higher in $A F-A B L$ than in $S R$ (adjusted HR $=2.57$, 95\% CI 1.09-6.02, $p=0.03$ ). What is more, no difference in long-term survival between $S R$ and $A F-n o n A B L$ was observed.

Conclusions: Despite the improvement of CRT pacing burden and thus response-rate up to the level of $A F$ subjects without the need for ablation, the long-term survival of AF patients requiring AVJ ablation remains still worse than in SR. (Cardiol J 2014; 21, 3: 309-315)

Key words: cardiac resynchronization, atrial fibrillation, heart failure, junction ablation

\footnotetext{
Address for correspondence: Ewa Jędrzejczyk-Patej, MD, Department of Cardiology, Congenital Heart Disease and Electrotherapy, Silesian Medical University, Silesian Center for Heart Diseases, ul. Skłodowskiej-Curie 9, 44-800 Zabrze, Poland, tel: +48 323733 682, fax: +48 323733 792, e-mail: ewajczyk@op.pl

Study data was presented at the $18^{\text {th }}$ World Congress on Cardiac Electrophysiology and Cardiac Techniques, Cardiostim 2012, June 13-16, Nice, France.
}

Received: 11.06.2013 Accepted: 18.06.2013 


\section{Introduction}

Cardiac resynchronization therapy (CRT) is an effective therapy in patients with symptomatic heart failure (HF), depressed left ventricular ejection fraction (LVEF), prolonged QRS complex and sinus rhythm (SR). It has been proven that CRT not only improves the functional capacity and quality of life, but also reduces mortality [1-5]. However, in a quite substantial percentage of $\mathrm{HF}$ patients CRT does not carry significant subjective and objective improvement [6]. Atrial fibrillation (AF) has been advocated as one of the potential factors linked to non-response in CRT recipients [7, 8]. This arrhythmia, which can be found in $30-40 \%$ of HF patients, contributes not only to the reduction of cardiac output but also to thromboembolic events. The profits of CRT in patients with AF have not yet been well established as majority of data on resynchronization therapy comes from trials performed in subjects with SR. As a consequence, the current European Society of Cardiology (ESC) guidelines do recommend CRT implantation in AF subjects only with a low class of recommendation and level of evidence [9].

The effectiveness of CRT may be compromised in $\mathrm{AF}$ patients by diminished percentage of effective biventricular pacing, especially in subjects with poor control of ventricular rate during arrhythmia. The ablation of atrioventricular junction (AVJ) is a potential solution in this subgroup, though data on the outcomes after this procedure are scarce. The aim of the study was to assess the impact of $\mathrm{AF}$ with and without the need for AVJ ablation on outcomes in HF patients treated with CRT.

\section{Methods}

\section{Study population, follow-up}

A single-center CRT registry has been designed to prospectively enroll and analyze 200 consecutive patients with first-time CRT device implantation. The study was conducted in a high volume tertiary care university hospital in Upper Silesia (a densely populated, urban area in southern Poland with over 4.6 million inhabitants). All included patients had drug - refractory symptomatic HF, New York Heart Association (NYHA) class III or IV, LVEF $\leq 35 \%$ and QRS duration $>120 \mathrm{~ms}$.

All subjects were routinely assessed 1 week and 1 month after the implantation and subsequently every 3-6 months. Clinical assessment, verification of medication and CRT check-ups (percentage of biventricular burden, electrical properties of leads, battery status and arrhythmic episodes) were performed at every visit.

Whenever the low resynchronization burden (percentage of biventricular pacing - BVP\% $<95$ ) was identified in an AF patient at any of the CRT check-ups, drugs slowing AV conduction (mainly beta-blockers and digoxin) were initiated or up-titrated to achieve maximal tolerable doses. Patients in whom despite optimal pharmacotherapy for at least 3-6 months BVP\% still remained low were qualified for AVJ ablation by the attending physician and electrophysiologist.

Prior to ablation, every patient was always informed in detail about the idea of the procedure and the consequent CRT dependency. Informed consent was obtained from all participants prior to their enrollment in the study. The study was complied with the Declaration of Helsinki and approved by the Ethical Committee.

\section{Implantation of CRT device, pacemaker settings and AVJ ablation}

Implantation of electrodes was performed in a standard way through a puncture of subclavian vein or using a cut-down technique. Right ventricular leads (or defibrillation electrode in patients receiving a cardioverter-defibrillator) were positioned in the apex. Left ventricular (LV) leads were implanted through the coronary sinus and lateral or posterolateral veins were preferred as target implantation sites. Implantation of atrial leads into a right-atrial appendage was performed in all subjects. Type of implanted electrodes (unipolar/bipolar, active/passive fixation) was left at the discretion of the operator. In SR patients CRT devices were set into DDD mode with a minimum heart rate of $60 \mathrm{bpm}$. In $\mathrm{AF}$ subjects a minimum pacing rate of $60-70 \mathrm{bpm}$ was set after the implantation. In patients who underwent AVJ ablation pacing rate was programmed at $80 \mathrm{bpm}$ for 3 weeks after the procedure, and reprogrammed thereafter to $60-70 \mathrm{bpm}$. At baseline antiarrhythmic functions of CRT were programmed in an identical way in all subjects. In each patient the AV (except for patients with permanent $\mathrm{AF}$ ) and interventricular delays were optimized under echocardiographic guidance in order to obtain consistent ventricular capture, maximal LV filling time without concomitant truncation of the $\mathrm{A}$-wave and to minimize inter- and intraventricular mechanical dyssynchrony $[10,11]$.

Radiofrequency AVJ ablation was performed in AF patients with low burden of CRT pacing if all attempts of drug therapy in order to slow the 
ventricular rate finally failed (usually with beta-blocking agent and digoxin), but not earlier than 1 month after CRT implantation. Ablation procedure was performed under local anesthesia by standard techniques in the electrophysiology cathlab. Two intracardiac electrodes were used: coronary sinus catheter (reference electrode used to confirm $\mathrm{AV}$ dissociation once conduction block had been created) and steerable ablation catheter. Acute success of AVJ ablation was $100 \%$, though in 4 patients the second procedure had to be performed after a mean of 2.5 months after the first ablation due to the recurrence of AV conduction.

\section{Analyzed data}

The following parameters were collected at baseline and after 6 months of follow-up (in AF subjects with AVJ ablation 6 months after the ablation): NYHA class, QRS duration (measured as the maximum QRS width in leads II, V1 and $\mathrm{V}_{6}$ ), $\mathrm{N}$-terminal pro B-type natriuretic peptide (NT-proBNP) level, echocardiographic parameters including end-diastolic and end-systolic volumes (EDV and ESV), as well as LVEF assessed with the use of Simpson's rule.

A positive clinical response to CRT was considered alive status without the need for heart transplantation and an improvement $\geq 1$ NYHA class after 6 months of pacing. The electrophysiologists who performed CRT device implantation and AVJ ablation were not engaged in assessment of NYHA class.

A positive echocardiographic response to CRT was considered alive status without the need for heart transplantation and $\mathrm{a} \geq 15 \%$ reduction in ESV after 6 months of pacing.

\section{Statistical analysis}

The continuous parameters were presented as median \pm range. Categorical variables were expressed as numbers and percentages. For continuous data intergroup differences were tested with the ANOVA with Kruskal-Wallis rang test. The $\chi^{2}$ test or Fisher's exact test with Bonferroni correction to control type I error was used to compare categorical values. The Kaplan-Meier survival and the log-rank test were used to compare survival probabilities. To assess the prognostic value of baseline data Cox regression analysis was performed, results of which were expressed as hazard ratio (HR) and 95\% confidence interval (CI). A p value of less than 0.05 was considered statistically significant. For multiple intergroup comparisons of baseline categorical parameters and response-rates, due to Bonferroni correction $\mathrm{p}$ values smaller than 0.017 were considered to indicate statistical significance for each of the $3 \mathrm{com}$ parisons. All statistical analyses were performed using the software package Statistica (version 6.0, StatSoft Inc., Tulsa, OK, USA and version 10.0).

\section{Results}

\section{Study population}

At the time of CRT device implantation 120 patients were in SR, whereas 80 subjects had AF (42 patients permanent and 38 paroxysmal form of AF). In 40 patients with $\mathrm{AF}$ and low BVP\% the AVJ ablation was performed after a mean of $5.5(2-16)$ months after CRT implantation. Finally, three groups of patients with CRT were analyzed: 1) AF with low BVP\% $<95 \%$ in which the successful AVJ ablation was performed (AF-ABL group, $\mathrm{n}=40,20 \%$ ), 2) $\mathrm{AF}$ without the need for AVJ ablation (BVP\% $\geq 95 \%$, AF-nonABL group, $\mathrm{n}=40,20 \%)$, 3) $\mathrm{SR}$ group $(\mathrm{n}=120,60 \%)$.

The study groups did not differ with respect to baseline characteristics, such as age, gender, ischemic etiology, QRS duration, NYHA class and echocardiographic parameters (Table 1). However, when compared to SR, AF-ABL group had significantly higher baseline NT-proBNP level (median 1471.0 vs. $2932 \mathrm{pg} / \mathrm{mL}, \mathrm{p}=0.046$ ).

Apart from significantly more frequent digoxin use in AF-ABL ( $p=0.0001$ vs. SR) and oral anticoagulants in AF patients, the study groups did not differ with respect to pharmacological therapy after CRT implantation. At follow-up visits a global biventricular percentage was similar among the three groups (BVP\% $\geq 95 \%)$.

\section{Response to CRT}

At 6 months of follow-up, a positive clinical response to CRT was demonstrated in $93(77.5 \%)$ subjects in SR group, 21 (52.5\%) in AF-ABL group $(\mathrm{p}=0.0025$ vs. SR group) and $20(50 \%)$ in AF-nonABL group ( $p=0.0009$ vs. SR group). There was no statistical difference in clinical response-rates between AF-ABL and AF-nonABL groups $(\mathrm{p}=0.82)$ (Fig. 1). A positive echocardiographic response to CRT was lower in AF-ABL group (19/40, 47.5\%) and AF-nonABL group $(21 / 40,52.5 \%)$ than in SR subjects $(72 / 120$, $60 \%)$, though the differences were not significant ( $\mathrm{p}=0.41$ for SR vs. AF-nonABL and $\mathrm{p}=0.17$ for SR vs. AF-ABL). Similarly, there was no statistically significant difference in echocar- 
Table 1. Baseline characteristics of study groups.

\begin{tabular}{|c|c|c|c|c|c|c|}
\hline & $\begin{array}{l}\text { Sinus rhythm } \\
\quad(\mathrm{n}=120)\end{array}$ & $\begin{array}{l}\text { AF-ABL } \\
(n=40)\end{array}$ & $\begin{array}{c}\text { AF-nonABL } \\
(n=40)\end{array}$ & $\mathbf{P}$ & $P^{\wedge}$ & P\# \\
\hline Age [years] & 59 (53-67) & $62(57-69)$ & $57(49-66)$ & 0.21 & 0.78 & 0.82 \\
\hline Female* & $32(26.7 \%)$ & $9(22.5 \%)$ & $9(22.5 \%)$ & 1.0 & 0.6 & 0.6 \\
\hline Diabetes mellitus type $2^{*}$ & $39(32.5 \%)$ & $16(40 \%)$ & $11(27.5 \%)$ & 0.24 & 0.39 & 0.56 \\
\hline Ischemic etiology* & $57(47.5 \%)$ & $19(47.5 \%)$ & $18(45 \%)$ & 0.82 & 1.0 & 0.78 \\
\hline QRS interval [ms] & 169.5 (155-185) & $160(140-190)$ & $177(160-200)$ & 0.2 & 0.85 & 0.72 \\
\hline NYHA IV* & $26(21.7 \%)$ & $14(35 \%)$ & $6(15 \%)$ & 0.04 & 0.09 & 0.36 \\
\hline LVEDV [mL] & $263(194-307)$ & 227 (164-278) & 237 (219-291) & 1.0 & 0.24 & 1.0 \\
\hline LVESV [mL] & 204 (147-244) & $172(116-204)$ & $186(171-218)$ & 0.65 & 0.15 & 1.0 \\
\hline LVEDD [mm] & 70 (66-79) & $69(64-77)$ & 74 (67-78) & 0.6 & 1.0 & 1.0 \\
\hline LVESD [mm] & $60(53-68)$ & $56(51-65)$ & $62(55-70)$ & 0.21 & 0.25 & 1.0 \\
\hline LVEF [\%] & $24(21-28)$ & $23.5(20-27.5)$ & $24(20-29)$ & 1.0 & 1.0 & 1.0 \\
\hline NT-proBNP [pg/mL] & $\begin{array}{c}1471.0 \\
(761.6-3323)\end{array}$ & $\begin{array}{c}2932 \\
(1390.5-4328)\end{array}$ & $\begin{array}{c}2410 \\
(889-4277)\end{array}$ & 1.0 & 0.046 & 0.795 \\
\hline Creatinine at admission $[\mu \mathrm{mol} / \mathrm{L}]$ & $94(71-117)$ & $98(82.5-117)$ & $91(78-110.7)$ & 0.89 & 0.795 & 1.0 \\
\hline \multicolumn{7}{|l|}{ Medication*: } \\
\hline Beta-blocker & $119(99.2 \%)$ & $39(97.5 \%)$ & $38(95 \%)$ & 0.56 & 0.41 & 0.09 \\
\hline ACE-I/ARB & $113(94.2 \%)$ & $37(92.5 \%)$ & $36(90 \%)$ & 0.69 & 0.71 & 0.37 \\
\hline Spironolactone & $116(96.7 \%)$ & $36(90 \%)$ & $35(87.5 \%)$ & 0.72 & 0.09 & 0.03 \\
\hline Loop diuretic & 109 (90.8\%) & $39(97.5 \%)$ & $38(95 \%)$ & 0.56 & 0.17 & 0.4 \\
\hline Digoxin & $25(20.8 \%)$ & $21(52.5 \%)$ & $9(22.5 \%)$ & 0.06 & 0.0001 & 0.82 \\
\hline Amiodarone & $26(21.7 \%)$ & $10(25 \%)$ & $15(37.5 \%)$ & 0.23 & 0.66 & 0.05 \\
\hline Vitamin $\mathrm{K}$ antagonists & $10(8.3 \%)$ & $33(82.5 \%)$ & $27(67.5 \%)$ & 0.12 & $<0.001$ & $<0.001$ \\
\hline
\end{tabular}

Continuous variables are presented as median (range); $\mathrm{ABL}$ — ablation; ACE-I/ARB — angiotensin-converting-enzyme inhibitors/angiotensin II receptor blockers; AF - atrial fibrillation; LVEDD - left ventricular end-diastolic diameter; LVESD - left ventricular end-diastolic diameter; LVEDV - left ventricular end-diastolic volume; LVESV - left ventricular end-systolic volume; LVEF — left ventricular ejection fraction; NT-proBNP - N-terminal pro B-type natriuretic peptide; NYHA - New York Heart Association classification; ${ }^{*} p<0.017$ was considered significant due to Bonferroni correction; $p-A F-A B L$ vs. AF-nonABL; ^ $p-S R$ vs. AF-ABL; \#p - SR vs. AF-nonABL

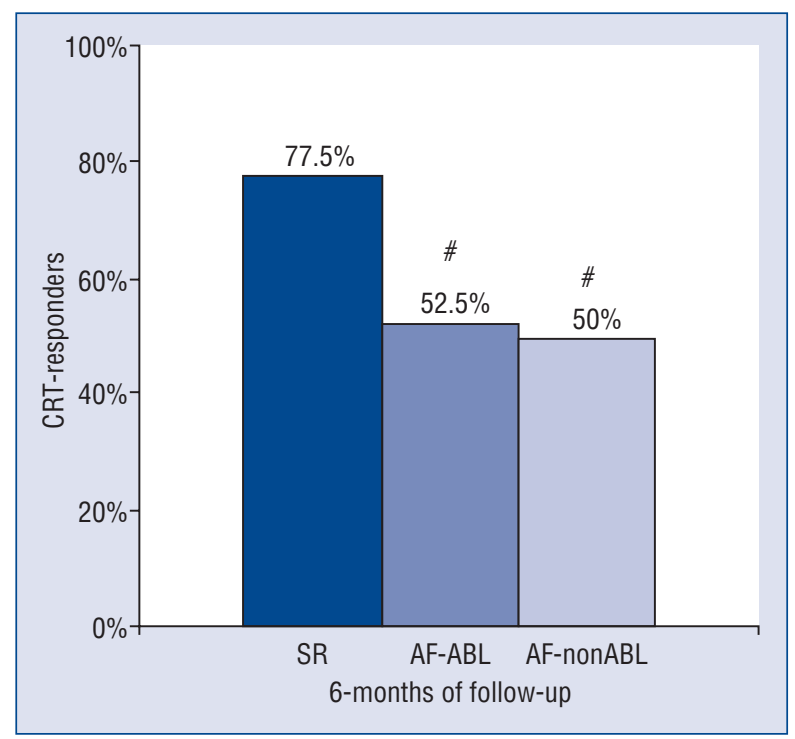

Figure 1. Percent of clinical cardiac resynchronization therapy (CRT)-responders in three groups of patients - with sinus rhythm and atrial fibrillation with and without the need for ablation (SR; AF-ABL; AF-nonABL); $\# p<0.017$ vs. sinus rhythm. diographic response-rate between $\mathrm{AF}-\mathrm{ABL}$ and AF-nonABL groups $(\mathrm{p}=0.65)$.

\section{Survival}

During a median follow-up of 36.1 (24.3-54.6) months the following number of patients died: $26(21.7 \%)$ patients in SR group, 13 (32.5\%) in AF-nonABL group and 11 (27.5\%) in AF-ABL group.

After adjustment for baseline confounder which was baseline NT-proBNP level, all-cause mortality was significantly higher in AF-ABL group than in SR group (adjusted HR $=2.57,95 \% \mathrm{CI}$ 1.09-6.02, $\mathrm{p}=0.03$ ), but did not differ between $\mathrm{SR}$ and $\mathrm{AF}-$ nonABL groups (adjusted $\mathrm{HR}=1.56$, 95\% CI 0.62-3.92, $\mathrm{p}=0.34$ ) (Fig. 2).

\section{Discussion}

According to the ESC guidelines CRT may be considered to reduce the risk of $\mathrm{HF}$ worsening in patients with $\mathrm{HF}$ and permanent $\mathrm{AF}$ who present with NYHA function class III or IV, LVEF $\leq 35 \%$ and $\mathrm{QRS}$ duration $\geq 120 \mathrm{~ms}$, who require pacing be- 


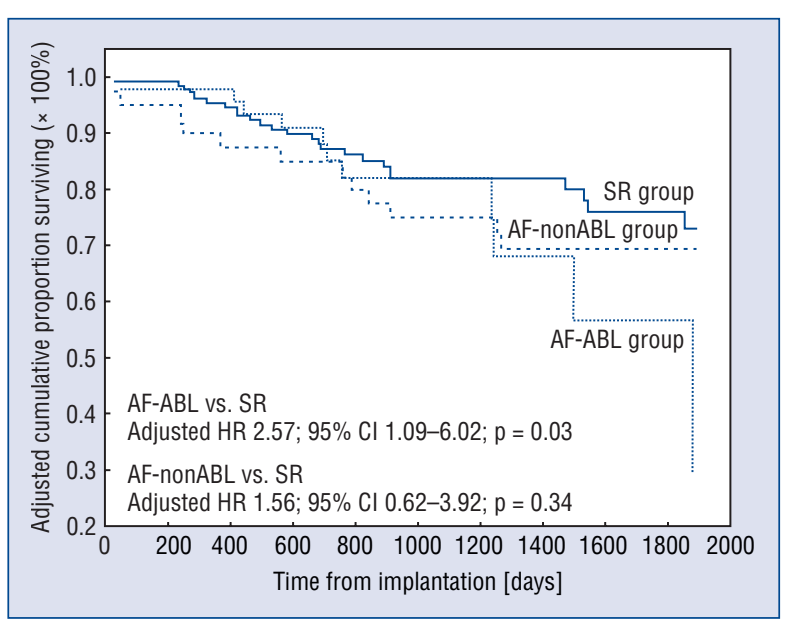

Figure 2. Kaplan-Meier curves of cumulative survival in patients with sinus rhythm and atrial fibrillation with and without the need for ablation (SR; AF-ABL; AF-nonABL). Survival curves were adjusted for baseline $\mathrm{N}$-terminal pro B-type natriuretic peptide level.

cause of an intrinsically slow ventricular rate or are CRT dependent as a result of AVJ ablation, or have ventricular rate $\leq 60 \mathrm{bpm}$ at rest and $\leq 90 \mathrm{bpm}$ on exercise [9]. However, there is still lack of randomized studies in patients with $\mathrm{AF}$ and data suggesting that these groups of patients may benefit from CRT come from observational studies. What is more, it has been even suggested that the benefit of CRT in AF subjects may be limited only to those patients who were subjected to AVJ ablation [12].

\section{Response to CRT in patients with SR and AF}

Our results indicate that the functional improvement achieved by resynchronization therapy may be similar in AF patients regardless of whether the ventricular rate can be adequately pharmacologically controlled or the AVJ ablation is required. Nevertheless, CRT response-rates of $\mathrm{AF}$ patients, irrespectively of the need for AVJ ablation, appeared to have been significantly worse when compared to subjects who were in SR.

Our results are concordant with other studies. Molhoek et al. [13] reported significantly lower percentage of responders in $\mathrm{AF}$ compared to the SR group (64\% vs. $80 \%, \mathrm{p}<0.05)$ and no statistical difference in AF-ABL vs. AF-nonABL groups (71\% vs. $54 \%, p=$ NS). Similarly, Tolosana et al. [14] observed comparable functional improvement after 12 months of CRT in AF-ABL and AF-nonABL sub- jects ( $58 \%$ vs. $59 \%$ CRT responders, $\mathrm{p}=\mathrm{NS}$ ) and a non-significant trend toward a lower percentage of responders in $\mathrm{AF}$ patients than in those with $\mathrm{SR}$ ( $59 \%$ vs. $70 \%, \mathrm{p}=\mathrm{NS}$ ). On the contrary, Ferreira et al. [15] documented a significantly lower percentage of responders among AF patients without AV node ablation when compared to those with SR, as well as those with $\mathrm{AF}$ and AVJ ablation (52\% vs. $79 \%$ vs. $85 \%, \mathrm{p}=0.008$ ).

The main difference between previously published data and our study is the qualification of AVJ ablation. Typically, $85 \%$ of pacing burden was used as a cut-off value for adequate biventricular pacing by other authors. However, according to the current ESC guidelines, the minimum value of $\geq 95 \%$ should be used as optimal one [9]. What is more, CRT burden of less than 95\% was associated with poor outcomes in several studies [16, 17]. Therefore, in line with current ESC guidelines, some patients from previously published trials would have presently had indications for AVJ ablation due to suboptimal CRT pacing burden. Furthermore, during a long-term follow-up, the percentage of biventricular pacing in AF-nonABL groups in the aforementioned studies still remained suboptimal and lower than in patients who underwent ablation $(87 \pm 19 \%$ vs. $98 \pm 6 \%$ in Ferreira et al. [15]; $82 \%$ vs. $100 \%$ in Molhoek et al. [13], and $92 \pm 7 \%$ vs. $100 \%$ in Tolosana et al. [14]). Of note is also that the $\mathrm{BVP} \%$ varied from $82 \%$ to $96.5 \%$ in $\mathrm{AF}$-nonABL subjects during a long-term follow-up [18]. Relatively liberal criteria that were used for AVJ ablation qualification and consequently suboptimal burden of CRT pacing in non-ablated group could in turn lead to the risk overestimation in these groups. In our study, AF patients were treated in line with the current ESC guidelines, and long-term pacing burden was similar in ablated and non-ablated AF groups, which diminished the intergroup heterogeneity and substantially facilitated the analysis of the gathered data.

\section{Survival}

Previously published data indicates that AF is an independent predictor of mortality in HF patients. In the Framingham Study, the development of $\mathrm{AF}$ in $\mathrm{HF}$ subjects was associated with significantly increased risk of death (1.6-fold in men and 2.7-fold in women) [19]. Also in CRT recipients it has been demonstrated than $\mathrm{AF}$ was an independent risk factor for death [20]. Tolosana et al. [21] documented a statistically significant higher probability of death from refractory HF at 12 months of follow up in patients with $\mathrm{AF}$ when compared to those 
who remained in SR (21\% vs. $5.7 \%, \mathrm{p}=0.005)$. Permanent AF was an independent predictor of mortality from HF in this study. Ferreira et al. [15] showed significantly lower survival in AF patients without $\mathrm{AV}$ node ablation compared with SR $(\mathrm{p}<0.001)$. In the same study the differences with respect to survival between SR vs. AF-ABL and AF-ABL vs. AF-nonABL were not significant ( $p=0.120$ and $p=0.070$, respectively). Gasparini et al. [12] observed similar mortality in SR and AF patients (HR 0.90, $\mathrm{p}=0.64$ ), whereas comparing AF-ABL with AF-nonABL groups, total mortality was significantly lower in the first one (adjusted HR 0.26, $p=0.010)$. Smit et al. [7] described the new-onset AF, permanent AF and NT-proBNP levels as independent predictors of mortality in CRT patients.

The main finding of our study is that despite AVJ ablation the long-term survival of AF patients is significantly worse than in SR group. Our data indicate that in $\mathrm{AF}$ patients with inadequate ventricular rate control AVJ ablation indeed leads to optimal CRT pacing burden and thus increase in the functional capacity and pre-specified response-rate up to the level of AF subjects without the need for ablation. However, the long term mortality in $\mathrm{AF}-\mathrm{ABL}$ group is still significantly higher than in SR group. Therefore, it seems that CRT recipients with AF may have similar outcomes to patients with SR, until their heart rate can be adequately pharmacologically controlled. The rationale for this phenomenon is unknown. However, AF with a fast ventricular conduction may induce tachycardiomyopathy and thus contribute to the aggravation of the preexisting HF, irrespectively of its primary etiology. Of note is also, that HF in this group of patients may already be so advanced and CRT introduced so late that despite optimal biventricular pacing the process of unfavorable remodeling can be neither slowed nor reversed.

Another open issue is, whether the rapid ventricular conduction during $\mathrm{AF}$ that requires AVJ ablation is a risk factor or just a marker of a more advanced disease (e.g. being a sign of a highly elevated sympathetic tone due to HF progression). Large, prospective, randomized trials are needed to address and answer these queries and finally assess the role of AVJ ablation in AF patients undergoing CRT.

\section{Limitations of the study}

Our study is a non-randomized, single-center observational analysis, with all the drawbacks typical for such designed studies. Relatively small study groups may have led to underestimation of some important relations and differences in baseline characteristics and pharmacological therapies which eventually could have created a bias. Another potential limitation may be differently programmed lower pacing rate in study groups, as well as the comparison of patients in SR with $\mathrm{AF}$ subjects regardless of the type of arrhythmia.

\section{Conclusions}

In conclusion, despite the improvement of CRT pacing burden and thus response-rate up to the level of AF subjects without the need for ablation, the long-term survival of AF patients requiring AVJ ablation still remains worse than in SR. A prospective, randomized clinical trial is necessary to assess the significance of AV node ablation in CRT-AF patients.

Conflict of interest: Ewa Jędrzejczyk-Patej: None; Radosław Lenarczyk: Received consultant fees from Medtronic and Biotronik; Patrycja Pruszkowska: None; Oskar Kowalski: Received consultant fees from Medtronic and Biotronik; Michał Mazurek: None, Adam Sokal: Received consultant fees from Medtronic and Biotronik; Joanna Boidol: None; Aleksandra Woźniak: None; Sławomir Pluta: None; Mariola Szulik: Received lecturer fee from GE Healthcare; Agnieszka Liberska: None; Zbigniew Kalarus: None

\section{References}

1. Anand IS, Carson P, Galle E et al. Cardiac resynchronization therapy reduces the risk of hospitalizations in patients with advanced heart failure: results from the Comparison of Medical Therapy, Pacing and Defibrillation in Heart Failure (COMPANION) trial. Circulation, 2009; 119: 969-977.

2. Cleland JG, Daubert JC, Erdmann E et al. The effect of cardiac resynchronization on morbidity and mortality in heart failure. N Engl J Med, 2005; 352: 1539-1549.

3. Linde C, Abraham WT, Gold MR, St John Sutton M, Ghio S, Daubert C.; REVERSE (REsynchronization reVErses Remodeling in Systolic left vEntricular dysfunction) Study Group. Randomized trial of cardiac resynchronization in mildly symptomatic heart failure patients and in asymptomatic patients with left ventricular dysfunction and previous heart failure symptoms. J Am Coll Cardiol, 2008; 52: 1834-1843.

4. Moss AJ, Hall WJ, Cannom DS et al. Cardiac-resynchronization therapy for the prevention of heart-failure events. N Engl J Med, 2009; 361: 1329-1338.

5. Tang AS, Wells GA, Talajic M et al. Cardiac-resynchronization therapy for mild-to-moderate heart failure. N Engl J Med, 2010; 363: 2385-2395.

6. Fornwalt BK, Sprague WW, BeDell P et al. Agreement is poor among current criteria used to define response to cardiac resynchronization therapy. Circulation, 2010; 121:1985-1991. 
7. Smit MD, Maass AH, Hillege HL, Wiesfeld AC, Van Veldhuisen DJ, Van Gelder IC. Prognostic importance of natriuretic peptides and atrial fibrillation in patients receiving cardiac resynchronization therapy. Eur J Heart Fail, 2011; 13: 543-550.

8. Borleffs CJ, Ypenburg C, van Bommel RJ et al. Clinical importance of new-onset atrial fibrillation after cardiac resynchronization therapy. Heart Rhythm, 2009; 6: 305-310.

9. McMurray JJ, Adamopoulos S, Anker SD et al. ESC guidelines for the diagnosis and treatment of acute and chronic heart failure 2012: The Task Force for the Diagnosis and Treatment of Acute and Chronic Heart Failure 2012 of the European Society of Cardiology. Developed in collaboration with the Heart Failure Association (HFA) of the ESC. Eur J Heart Fail, 2012; 14: 803-869.

10. Mele D, Pasanisi G, Capasso F et al. Left intraventricular myocardial deformation dyssynchrony identifies responders to cardiac resynchronization therapy in patients with heart failure. Eur Heart J, 2006; 27: 1070-1078.

11. Vanderheyden M, De Backer T, Rivero-Ayerza M et al. Tailored echocardiographic interventricular delay programming further optimizes left ventricular performance after cardiac resynchronization therapy. Heart Rhythm, 2005; 2: 1066-1072.

12. Gasparini M, Auricchio A, Metra M et al. Long-term survival in patients undergoing cardiac resynchronization therapy: The importance of performing atrio-ventricular junction ablation in patients with permanent atrial fibrillation. Eur Heart J, 2008; 29: 1644-1652.

13. Molhoek SG, Bax JJ, Bleeker GB et al. Comparison of response to cardiac resynchronization therapy in patients with sinus rhythm versus chronic atrial fibrillation. Am J Cardiol, 2004; 94: 1506-1509.

14. Tolosana JM, Hernandez Madrid A, Brugada J et al. Comparison of benefits and mortality in cardiac resynchronization therapy in patients with atrial fibrillation versus patients in sinus rhythm. (Results of the Spanish Atrial Fibrillation and Resynchronization [SPARE] Study). Am J Cardiol, 2008; 102: 444 -449.

15. Ferreira AM, Adragao P, Cavaco DM et al. Benefit of cardiac resynchronization therapy in atrial fibrillation patients vs. patients in sinus rhythm: the role of atrioventricular junction ablation. Europace, 2008; 10: 809-815.

16. Hayes DL, Boehmer JP, Day J et al. Cardiac resynchronization therapy and the relationship of percent biventricular pacing to symptoms and survival. Heart Rhythm, 2011; 8: 1469-1475.

17. Koplan BA, Kaplan AJ, Weiner S, Jones PW, Seth M, Christman SA. Heart failure decompensation and all-cause mortality in relation to percent biventricular pacing in patients with heart failure: Is a goal of 100\% biventricular pacing necessary? J Am Coll Cardiol, 2009; 53: 355-360.

18. Ganesan AN, Brooks AG, Roberts-Thomson KC, Lau DH, Kalman JM, Sanders P. Role of AV nodal ablation in cardiac resynchronization in patients with coexistent atrial fibrillation and heart failure: A systematic review. J Am Coll Cardiol, 2012; 59: 719-726.

19. Wang TJ, Larson MG, Levy D et al. Temporal relations of atrial fibrillation and congestive heart failure and their joint influence on mortality: The Framingham Heart Study. Circulation, 2003; 107: 2920-2925.

20. Wilton SB, Leung AA, Ghali WA, Faris P, Exner DV. Outcomes of cardiac resynchronization therapy in patients with versus those without atrial fibrillation: A systematic review and meta-analysis. Heart Rhythm, 2011; 8: 1088-1094.

21. Tolosana JM, Arnau AM, Madrid AH et al. Cardiac resynchronization therapy in patients with permanent atrial fibrillation. Is it mandatory to ablate the atrioventricular junction to obtain a good response? Eur J Heart Fail, 2012; 14: 635-641. 